\title{
Videoconferencia en la educación
}

\section{Videoconferencing in education}

Emilio A. Rivera Landero ${ }^{\text {. }}$

\begin{abstract}
:
The use of videoconferencing systems in the field of education has had an increase during the period of March 2020 until today due to the pandemic caused by COVID-19 that forced schools to implement distance education, however, it was clear that not all teachers had the skills to develop sessions on these platforms, coupled with the lack of knowledge about the characteristics of each one; in this essay, aspects to consider when thinking about a virtual didactic strategy are cited, as well as elements of videoconferencing platforms in general and specifically, 10 characteristics of the Zoom, Meet and Teams applications are compared; which allows to guide decisions when implementing them as part of a correct instructional design.
\end{abstract}

Keywords:

Videoconferencing, education, educational technology

\section{Resumen:}

El uso de los sistemas de videoconferencia en el ámbito de la educación ha tenido durante el periodo de marzo de 2020 a la fecha un aumento debido a la pandemia ocasionada por el COVID-19 que obligó a las escuelas a implementar una educación a distancia, sin embargo, resultó claro que no todos los docentes contaban con las competencias para desarrollar sesiones en estas plataformas aunado a la falta de conocimiento sobre las características de cada una; por lo que en el presente ensayo se citan aspectos a considerar al pensar en una estrategia didáctica virtual así como elementos de las plataformas de videoconferencia en general y de forma específica, se comparan 10 características de las aplicaciones Zoom, Meet y Teams; lo que permite orientar las decisiones al implementarlas como parte de un correcto diseño instruccional.

\section{Palabras Clave:}

Videoconferencias, educación, tecnología educativa

\section{Introducción}

La etapa de pandemia de la que nos ha tocado ser testigos y actores, ha puesto a prueba a toda la sociedad y de manera particular a las Instituciones educativas y dentro de ellas a los docentes, quienes han tenido que utilizar materiales y recursos didácticos que antes de esta etapa no habrían considerado en una educación presencial, por lo que "el uso de estos elementos en formato digital han representado un verdadero desafío." (García, 2020)

De entre dichos recursos didácticos digitales, se encuentra el de las videoconferencias, como una forma de suplir las sesiones presenciales así como para asesorías y tutorías, ya que de acuerdo a Ramos (2020) con base en datos proporcionados por el reporte Digital Consumer Trends en México 2020, "el comportamiento de consumo digital en México y el impacto del COVID-19 se muestra que actualmente $46 \%$ de los mexicanos hace uso de las plataformas de videoconferencias", para diversas actividades entre ellas las educativas.

En el presente trabajo se identifica en un primer momento, la diferencia entre un material y un recurso instruccional, además de la manera en que se articulan dentro de una estrategia de enseñanza, para posteriormente revisar los sistemas de videoconferencias como recursos a utilizar para desarrollar dichas estrategias, señalando sus características generales, ventajas, desventajas y ejemplos de los sistemas comerciales actualmente disponibles.

Finalmente se presentan una serie de conclusiones sobre la implementación de las videoconferencias como recurso de enseñanza.

Autor de Correspondencia, Universidad Autónoma del Estado de Hidalgo, https://orcid.org/0000-0002-8685-3746, Email: rlandero@uaeh.edu.mx 


\section{Desarrollo}

Durante el proceso de enseñanza-aprendizaje los docentes utilizan, materiales y recursos de enseñanza en el contexto de una estrategia de enseñanza.

Fernández Lomelí (2011) indica la diferencia entre un material y un recurso didáctico al señalar que estos términos no se refieren a lo mismo, ya que el material didáctico es un objeto, que puede ser físico o virtual, el cual se utiliza con el objetivo de facilitar la construcción del aprendizaje mientras que el recurso didáctico incluye técnicas y materiales que buscan desarrollar aprendizajes significativos.

Siguiendo a Fernández Lomelí (2011) se identifica que un recurso se convierte en didáctico cuando se utiliza precisamente para promover aprendizaje, de tal forma que un simulador o el "uso de internet, entre otros [...] puede ser un recurso didáctico" (Fernández Lomelí, 2011, pág. 13) cuando se emplea con el objetivo de que los alumnos desarrollen un determinado aprendizaje.

Por su parte, Castañeda Yáñez (2004) identifica los medios de comunicación a los cuales tenemos acceso tanto de forma física como electrónica, como recursos que pueden volverse instruccionales y que permiten al alumno contar con una experiencia de la realidad que ella denomina "indirecta". Para su uso instruccional se debe considerar tanto la parte intelectual, es decir la organización didáctica, como el aspecto mecánico, esto es, el equipo que se utiliza para llevar a cabo este proceso, así como el lenguaje a utilizar: verbal, visual, escrito o de tipo multimedia.

Dado que los materiales y recursos didácticos deben utilizarse en el marco de una estrategia de enseñanza, a continuación se revisarán elementos básicos para entenderla. Con esta finalidad se seguirá la siguiente definición para estrategia de enseñanza entendiéndola como "procedimientos que el agente de enseñanza utiliza de forma reflexiva y flexible para promover el logro de aprendizajes significativos en los alumnos" (Díaz-Barriga Arceo y Hernández Rojas, 2010, pág. 118)

Díaz-Barriga Arceo y Hernández Rojas (2010) clasifican las estrategias de la siguiente manera:

- Para activar y usar los conocimientos previos y para generar expectativas apropiadas en los alumnos.

- Para mejorar la integración constructiva entre los conocimientos previos y la nueva información por aprender.

- Discursivas y enseñanza.

- Para ayudar a organizar la información por aprender.

- $\quad$ Para promover una enseñanza situada.

Díaz-Barriga Arceo y Hernández Rojas (2010) aclaran que las estrategias citadas, si bien son pensadas para la educación presencial, también pueden aplicarse en la educación semipresencial (b-learning) o virtual (elearning). Por lo tanto es necesario que el diseño de una buena estrategia incluya los medios, recursos y materiales.

Ahora bien, ¿que recursos utilizar dentro de una estrategia que se encuentra inmersa en el b-learning? Para contestar a esta interrogante se requiere pensar en recursos digitales basados en las Tecnologías de la información y la comunicación (TIC) que se encuentran al alcance de alumnos y docentes. Sin embargo hacer esta selección no es sencillo, es una tarea que requiere de un trabajo de profunda reflexión y conocimiento por parte de los docentes y para la cual se requiere contar con una aceptable alfabetización tecnológica, ya que "una persona tecnológicamente alfabetizada está capacitada para comprender las aplicaciones de las tecnologías y las decisiones que implican su utilización" (Azinian, 2009)

Así pues, el docente que implementa el uso de tecnología en la clase requiere relacionarse con dicha tecnología desde un punto de vista que le permita comprender el alcance de la misma y sus limitaciones, así como la forma en que puede aprovecharla de forma estratégica dentro del proceso de enseñanza aprendizaje.

En tal sentido y aun cuando en este momento el uso de los sistemas de videoconferencia se ha convertido en una práctica cotidiana, se requiere realizar una revisión de las particularidades de dichos sistemas para su aplicación en la educación a distancia tanto e-learning como b-learning. Sin olvidar que existen otros sistemas basados en internet que permiten la comunicación, como el caso de los correos electrónicos, los foros de discusiones y redes sociales; se centrará la atención en los sistemas de Videoconferencia por ser estos los que ofrecen una experiencia más cercana al aula de clase y la comunicación con grupos en tiempo real.

Una Videoconferencia es el sistema que "permite establecer una comunicación sincrónica, recibiendo y enviando señales de audio y video de una computadora a otra, mediante la utilización de un software, o a través de algún servicio web." (Vidal Martínez y Aguilar Valenzuela, 2014)

En este punto se aclara que, si bien en algunos espacios se utiliza el término de Videoconferencia de escritorio para referirse a los sistemas donde solo se requiere el uso de una computadora conectada a la red con elementos básicos como un micrófono y cámara web para mejorar la experiencia, o bien un dispositivo de comunicación móvil como celular o tableta; en el presente trabajo únicamente se utilizará el término Videoconferencia refiriendo el ambiente técnico recién descrito.

Las aplicaciones de las Videoconferencias son múltiples sin embargo en donde actualmente tienen mayor uso de 
acuerdo con (Vidal-Saucedo y Álvarez-Mariaud, 2021) son:

- Instituciones educativas (escuelas, universidades, centros de capacitación, etcétera)

- $\quad$ Empresas privadas

- Sector industrial

- Dependencias gubernamentales y de servicios

- Instituciones financieras (bancos, casas de bolsa entre otras)

- Sector salud (hospitales, clínicas, centros de salud, etcétera)

- Sector turístico (cadenas hoteleras, agencias de viajes, compañías de transportación, entre otros).

Es importante hacer notar que estos sistemas tienen ya varios años que son implementados en ambientes como los citados por Vidal-Saucedo y Álvarez-Mariaud (2021), entre ellos en la educación, tal como lo comenta Reinoso González (2020) cuando cita que "La videoconferencia es una herramienta de enseñanza efectiva con evidencia sólida de su implementación en educación superior universitaria", sin embargo ha sido notable el crecimiento de su implementación en los últimos meses.

Con base en la experiencia en el uso de estos sistemas es posible identificar algunas de sus ventajas como son:

- $\quad$ Se encuentra basada en internet

- Fomenta la comunicación en las dos vías, tanto en audio y video como por mensajes.

- Flexible para cualquier tipo de estrategia a implementar

- $\quad$ Permite generar buenas experiencias

- $\quad$ Facilita mantener la motivación del estudiante

- Es posible utilizarlo en tutorías y asesorías

- $\quad$ Permite la aplicación de evaluaciones

- $\quad$ Permite calendarizar las reuniones.

- Posibilita compartir la pantalla de los equipos y realizar anotaciones en pizarras electrónicas.

- $\quad$ Se accede a distintos tipos de vista de la misma sesión.

Como todas las herramientas y sistemas, se tienen desventajas o limitaciones, entre ellas "que la calidad de la comunicación depende del ancho de banda del enlace [...] la saturación que tenga la red, así como las características físicas de los equipos en ambas partes" (Vidal Martínez y Aguilar Valenzuela, 2014)

Durante su implementación es necesario considerar aspectos que el docente y el alumno deben cubrir tal como lo mencionan Isla Montes y Ortega Molina (2001), entre los cuales están por parte del docente: 1) su formación y entrenamiento, 2) el material a utilizar, 3) la materia, 4) los métodos de evaluación, 5) un sistema de asesorías y 6) el control del aula virtual.
En cuanto al alumno, entre los aspectos que deben tomarse en cuenta se encuentran: 1) la motivación, 2) su participación, 3) el aprendizaje, 4) el sentido de pertenencia al grupo y 6) su experiencia en el uso de dichas herramientas.

\section{Sistemas de videoconferencia.}

En relación a los sistemas disponibles de forma comercial, actualmente se cuenta con una amplia gama de sistemas de videoconferencia entre las cuales se encuentran Zoom, Meet y Teams. De los cuales se presenta a continuación una descripción de sus características, considerando los siguientes elementos: 1) Empresa que lo desarrolla, 2) Costo, 3) Número de participantes en una videoconferencia, 4) Duración de las sesiones, 5) Posibilidad de trabajar en equipos dentro de la sesión, 6) Desarrollo de Webinars, 7) Registro de asistentes, 8) Grabar sesiones, 9) Transmisión a redes sociales, 10) Crecimiento en 2020.

- Zoom. Aplicación creada por Zoom Video Communications Inc., cuenta con oferta gratuita y varias opciones con pago que hacen crecer las facilidades del programa, de esta forma en el plan gratuito se tienen sesiones con una duración máxima de 40 minutos y 100 participantes, mientras que en la versión de paga mas accesible las conferencias no tienen límite de tiempo pero solo para 100 participantes pudiendo incrementar el número de participantes a 1000 si se adquiere un complemento. Zoom permite crear salas con equipos pequeños lo que para una sesión de clase es muy conveniente si se desea que los alumnos del grupo trabajen en pequeños grupos dentro de la misma sesión. Esta aplicación permite la creación de Webinars lo que posibilita la realización de eventos en línea con vídeo, audio y pantalla compartida con hasta 100 participantes de vídeo y 10000 asistentes. Zoom mantiene un registro de asistentes a las sesiones y la consulta de las personas que asisten a las videoconferencias lo que facilita a un docente automatizar el pase de lista. Las sesiones en esta aplicación pueden ser grabadas siempre que se cuente con un plan de pago así como transmitir la sesión en vivo a redes sociales como Facebook y YouTube. (Zoom, 2021) De acuerdo a Ramos (2021) la plataforma fue la de mayor crecimiento en 2020 pasando de 10 millones de usuarios en diciembre de 2019 a 300 millones en abril de 2020.

- Meet. Es una solución gratuita de Google que sustituyó a Hangouts, por lo que se encuentra vinculado con Gmail y el resto de soluciones de Google (Workspace). Cuenta con varias ediciones y de acuerdo a la edición con se cuente pueden variar las opciones disponibles, la edición básica no tiene costo. En las 
videoconferencias pueden participar desde 100 y hasta 250 personas dependiendo la edición con que se cuente y no tienen límite en el tiempo de duración. Permite crear grupos de trabajo para dividir a los participantes en grupos más pequeños. Las versiones de pago cuentan con la característica de un registro de asistencia y es posible grabar una sesión siempre que el administrador de Google Workspace lo active para la cuenta. Actualmente no es posible enviar la señal desde la plataforma para ser compartida a una red social, (Google, 2021). Ramos (2021) indica que esta plataforma cuenta con 3 millones de nuevos usuarios cada día en el mundo.

- $\quad$ Teams. Es la propuesta de Microsoft, por lo que es posible vincular al resto de servicios de dicha compañía como Office 365 , no tiene costo y únicamente se requiere contar con una cuenta de Microsoft y/o agregar un número de teléfono o bien optar por Office365 para el ámbito educativo. Tiene un límite de 100 personas en una sesión y en cuanto al tiempo el límite que contempla es de 24 horas. Para crear salas durante una sesión se requiere contar con la versión de escritorio ya sea para Windows o Mac, mientras que para habilitar un Webinar o eventos en directo los límites son de 10,000 asistentes por 4 horas, La plataforma también permite descargar un informe de asistencia en formato CSV (que puede abrirse con Excel) con el nombre de cada persona en la reunión y la hora en que se unieron y salieron de la reunión. Las sesiones pueden ser grabadas capturando audio, vídeo y actividad de uso compartido de pantalla pero no la pizarra. Actualmente no es posible transmitir las sesiones a una red social como Facebook, (Microsoft, 2021). En cuanto al número de usuarios Teams pasó de "32 millones en marzo 2019 [a] 75 millones en abril 2020" (Ramos, 2021)

Finalmente, optar por uno $u$ otro de los sistemas de videoconferencias, si bien debe estar guiada por razones académicas es cierto que también influyen aspectos económicos, de preferencia personal e incluso de decisiones institucionales. En cualquier caso, es obligatorio conocer y practicar con la herramienta seleccionada antes de implementarla como apoyo para las sesiones de clase, de asesoría y/o de tutoría.

\section{Conclusiones}

Al iniciar el periodo de pandemia los docentes se vieron obligados a implementar tecnología para sus sesiones de clase y sin mucho tiempo para realizar un análisis profundo sobre los sistemas a implementar, el uso de los sistemas de videoconferencia se convirtió en una práctica cotidiana, con aciertos y errores que se identificaron mediante prueba y error, lo que ha ocasionado experiencias positivas pero también negativas.
Los sistemas de videoconferencia pueden ser integrados de forma exitosa en los modelos de educación b-learning así como e-learning, por lo que es necesario que el docente conozca y compare las características, alcances y limitaciones de dichos sistemas para que haga uso de ellos dentro de una estrategia de enseñanza enmarcada en un correcto diseño instruccional, de tal manera que se potencialicen las bondades de los mismos con el objetivo de generar aprendizajes significativos.

Queda mucho por recorrer y aprender así como retos a superar en el camino de las videoconferencias en la educación, pero sin duda, la experiencia ganada en los últimos meses representa una base para el trabajo futuro.

\section{Referencias}

Azinian, H. (2009). Las tecnologías de la información y la comunicación en las prácticas pedagógicas (Primera ed.). Buenos Aires, Argentina: Novedades educativas.

Castañeda Yáñez, M. (2004). Los medios de la comunicación y la tecnología educativa (Segunda ed.). México: Trillas.

Díaz-Barriga Arceo, F., Hernández Rojas, G. (2010). Estrategias docentes para un aprendizaje significativo. Una interpretación constructivista (Tercera ed.). México: McGraw Hill.

Fernández Lomelí, A. G. (2011). Recursos didácticos, elementos indispensables para facilitar el aprendizaje. México: Limusa.

García L. (2020) Coronavirus. Educación y uso de tecnologías en días de pandemia. El desafío de modificar las estrategias pedagógicas y de las brechas digitales que limitan el acceso a la educación. Recuperado el 1 de septiembre de 2021, de Ciencia UNAM: http://ciencia.unam.mx/leer/1006/educacion-y-uso-de-tecnologias-endias-de-pandemia

Google. (2021). Google Meet. Recuperado el 25 de Junio de 2021, de Google Meet: https://apps.google.com/meet/

Isla Montes, J. L., Ortega Molina, F. D. (2001). Consideraciones para la implantación de la videoconferencia en el aula. Pixel-Bit. Revista de Medios y Educación (17), 23-31.

Microsoft. (2021). Teams. Recuperado el 25 de Junio de 2021, de Teams: https://www.microsoft.com/es-mx/microsoft-teams/group-chatsoftware

Ramos M. (2021) Plataformas de videoconferencias durante el confinamiento: hasta $300 \%$ de crecimiento en 2020. Recuperado el 21 de septiembre de 2021 de Marketing ecommercemx: https://marketing4ecommerce.mx/las-plataformas-devideoconferencias-durante-el-confinamiento/

Reinoso González, E. (25 de Mayo de 2020). La videoconferencia como herramienta de educación: ¿qué debemos considerar? Revista española de educación médica, 60-65.

Vidal Martínez, A. A., Aguilar Valenzuela, F. (2014). La Videoconferencia de escritorio como una herramienta para el desarrollo. Actualidades Investigativas en Educación, 14(2), 1-21. 
Publicación semestral, Ingenio y Conciencia Boletín Científico de la Escuela Superior Ciudad Sahagún, Vol. 9, No. 17 (2022) 30-34

Vidal Saucedo, L. E., Álvarez Mariaud, C. (2021). EDUCREA. Recuperado el 24 de Junio de 2021, de Videoconferencia: Herramienta para la educación no presencial: https://educrea.cl/videoconferenciaherramienta-para-la-educacion-no-presencial/

Zoom. (2021). Zoom. Recuperado el 25 de Junio de 2021, de Zoom: https://zoom.us 